\title{
Orchestrating a Network of Activities in the Value Chain
}

\author{
$\mathrm{N}$ Viswanadham and S Kameshwaran \\ Center for Global Logistics and Manufacturing Strategies, \\ Indian School of Business, \\ Hyderabad 500032 \\ Email: N_Viswanadham@isb.edu, Kameshwaran_S@isb.edu
}

\begin{abstract}
The orchestrator is a management literature metaphor to describe the role of a player who organizes and manages a set of activities in a network, by ensuring valuecreation opportunities in the system and value appropriation mechanisms for each player. In this paper, we consider orchestrators who do not own capacities but have access to a large pool of globally dispersed service providers in the various stages of the supply chain. For a given customer order, the orchestrator identifies the right set of service providers and coordinates the entire execution such that order is delivered as per the requirements. We propose a mixed integer program to optimally choose the service providers taking into account the capacities, production and distribution costs, international taxation, tariffs, and coordination costs.
\end{abstract}

\section{INTRODUCTION}

There are three innovation business models: integration, orchestration, and licensing [1]. Integrators perform all the activities in the value chain and retain maximum control over every aspect of execution process. Orchestrators allow some or all of the activities to be performed by other players. In licensing, companies achieve payback on their ideas and intellectual assets without having to make an investment in commercialization or realization. At times, companies can use all the three models depending the product or service being offered and the market conditions. Our focus in this paper is orchestration, the popular model of innovation collaboration.

The orchestrator is a management literature metaphor to describe the role of a player who organizes and manages a set of activities in a network, by ensuring value-creation opportunities in the system and value appropriation mechanisms for each player [2]. Orchestration brings about and manages whole set of tangible and intangible elements starting from design to distribution. Unlike outsourcing, orchestrators manage a network of contributors who have a stake in the outcome. Microsoft orchestrated different lines of Tablet PCs in partnership with hardware companies like IBM, HP, Toshiba, Acer, and Fujitsu. Bath \& Body Works partnered with suppliers of specialty goods including C. O. Bigelow, L'Occitane, Goldie, and American Girl, to provide new lines of beauty and personal care products. The automotive and aviation industries, which are traditionally integrators, also moved towards orchestration: BMW partnered for the creation of active steering system and Boeing partnered with external players to provide various integral parts to the aircraft 787. For a comprehensive discussion on the reasons, paybacks, and the risks in orchestration, see [1].

There are also companies that orchestrate for others. Medion AG (Germany) orchestrates the entire value chain from the initial product to after-sales services of computers and peripherals for its retail customers [3]. Li \& Fung (Hong Kong) is a trading company that provides it clients with a virtual company for manufacturing apparels and toys [4], [5]. The clients, usually from US and Europe, approach Li \& Fung with demand for a certain apparel. The company has a network of thousands of globally dispersed service providers. Based on the client requirements, service providers are chosen to provide each of the constituent activities, thus creating a virtual company. Li \& Fung owns no factories or hard capacities and all the activities in the value creation, except for coordination, is performed by other service providers. In this paper, we propose a mixed integer program for orchestrating a network of activities by companies like $\mathrm{Li}$ $\&$ Fung.

The rest of the paper is organized as follows. Section II describes in detail the orchestration of globally dispersed network of activities for a customer. The related literature from the design of global supply chains that are relevant to the orchestration is briefly reviewed in Section III. The model is mathematically presented and an optimization model is proposed in Section IV for orchestration. Section V concludes the paper with possible future directions of research.

\section{Orchestrating A Network of Activities}

The orchestrator that is considered in this paper is largely motivated by Li \& Fung (Trading) Ltd. of Hong Kong [4], [5]. It primarily operated as an agent, finding suppliers to manufacture items according to customers' specifications. The items included garments, toys, household items, sporting goods, handicrafts, and fashion accessories. The company was divided into several dozen independent divisions, each of which concentrated on orchestrating for one category of products and served one or few customers. The company has a international sourcing network with thousands of suppliers in over dozens of countries. Different countries offered different combinations of manufacturing capabilities, quality standards, and cost. The international sourcing network is not a formally constituted entity but consists of two intangible assets: relationships with the service providers and knowledge of the manufacturing capabilities, special skills, business practices, and regulations pertaining to each 
country and each supplier. The knowledge also includes the hidden costs like tariffs, duties, taxes, quotas, customs declaration processes, security requirements, and interfacing with government authorities. Further, all these are frequently subject to change. Li \& Fung's business depends on these assets which leverages the international differences in labor costs and manufacturing capabilities to provide products that closely matches the customers' requirements with respect to price, quality, and delivery time.

The following example illustrates how a network of activities are orchestrated when a typical order is received at Li \& Fung [6]. Consider an order for 10,000 garments from a European retailer. The yarn will be sourced from Korea, but woven and dyed in Taiwan. The zippers are ordered from China and because of labor conditions and quotas, the garments will be made in five factories in Thailand. Finally, the garments will be shipped from Thailand to Europe all arriving in identical condition, as if made in a single integrated factory. The total lead time is five weeks, since the order was placed. Following features can be inferred from above illustrative example (few of them have been identified for Medion AG in [3]).

\section{A. Build-to-order}

The supply chain is build-to-order and the customer's order initiates the design, procurement, production, and distribution.

\section{B. Zero Inventory}

The planning horizon is single period and the demand is to be realized in near future (few weeks). Thus there is zero inventory to be maintained at any stage.

\section{Value Chain Disintegration}

The entire value chain is dissected into a chain of activities and each can possibly be provided by different service providers - chosen and coordinated by the orchestrator such that the entire execution is optimized.

\section{International Sourcing and Division of Labor}

The service providers are dispersed globally and the costs of trading in a country is governed by various economic and foreign trade policies. Further, there are issues related to international trade and tariffs that are to be taken into account.

\section{E. Project-based Activity}

Each order from the customer is considered as a single project. Each project is managed by a team which ensures that all the required production steps are integrated, completed on time, and the products have met quality specifications. Coordination costs need to be considered, especially when working with new service providers.

\section{F. Service-oriented}

The orchestration process is a service-oriented activity though the deliverable is a manufactured product. The challenges faced by the orchestrator in order to be competitive in the market are sustaining relationship with the service providers, developing new relationships, updating their skills, updating the knowledge of the international regulations, and adapting its own governance structure, according to market conditions.

\section{G. Optimization Problem}

The problem of choosing the service providers for each activity is clearly an optimization problem. This is a typical supply chain design problem that addresses issues related to supplier selection, order allocation, facility location, and capacity planning. These are generally strategic and tactical decisions in traditional supply chains. But in this case, with the single period planning horizon of just few weeks, the decision problem is more operational in nature. In the next section, we review briefly the literature related to global supply chain design.

\section{Global Supply Chain Design}

In the last two decades, supply chains expanded globally, especially in automobile, computer, consumer electronics, and apparel industries. A supply chain design problem comprises the decisions regarding the number and location of production facilities, the amount of capacity at each facility, the assignment of each market region to one or more locations, and supplier selection for sub-assemblies, components and materials [7]. In global supply chain design, suppliers, production facilities, distribution centers, and markets can be at international locations. The major factors that affect the decision making in international sourcing and division of labor are exchange rates, duties and duty drawbacks, non-tariff trade barriers, international taxation, and transfer prices.

\section{A. Factors due to Globalization}

Currency exchange rates affect the price paid for goods that are purchased in the supplier's currency. A simple example based on real exchange rate movements in [8] shows that a international production network can witness a decrease of profits by almost 50\%. Three types of currency exposures are identified due to exchange rate uncertainties [9]:

- Accounting exposure refers to the effects currency fluctuations can have on consolidated financial statements.

- Transaction exposure refers to the effects on contractually defined cash flows.

- Operational exposure refers to the change of the present value of a firm resulting from changes to future operating cash flows caused by unexpected currency fluctuations.

The first two types of currency exposures are controlled by currency hedging, while the operational exposure requires 
operational hedging with the flexibility of re-allocating production within the network.

Duties are import taxes levied at the border for importing a product into the country. Duties are used as means of protecting the domestic companies and also as a revenue for the country. Free trade agreements allow participating countries to trade without any duties and have the same duty structure for a non-participating country to all the countries in the agreement. Duties are usually calculated as a percentage of the value of the imported goods including the cost of shipping. Duty drawbacks are allowed if the imported goods are re-exported in the identical condition or in a different condition. Network design can help in choosing the appropriate locations for different activities such that the company can gain by avoiding duties and also by leveraging duty drawbacks.

Non-tariff trade barriers are another form of protecting domestic companies. The most common ones are local content rules (certain amount of purchasing has to be done from local suppliers) and import quotas (restriction on quantities of goods that can be imported). Local content rules are more prevalent in automobile industry and usually affect the sourcing of raw materials and sub-assemblies. Import quotas are generally implemented by developed countries to restrict the import from developing countries and affect the production decision of the finished product.

International tax regimes and transfer prices significantly influence structure of the global supply chains. Transfer pricing refers to price of intermediate goods and services exchanged between the various stages in the supply chain. In order to incorporate taxes and transfer pricing into supply network design, the after-tax profit maximization is usually used as the objective.

\section{B. Relevance to Orchestration}

The supply chain design problem faced by the orchestrator considered in this paper is similar in spirit to global supply chain design, but only few of the above factors are relevant. The orchestrator is assumed to own no facility and hence there are no transfer prices. The trade with service providers are through external price transactions, which will also include the appropriate taxes. Thus taxes need not be included exclusively. The currency exchange rate fluctuations only lead to only accounting and transaction exposures as the planning horizon is relatively short. As these exposures can be handled through currency hedging, they do not play significant role in supply chain design (in terms of operational flexibility and extra capacity). However duties, duty drawbacks, and import quotas have to be taken into account and are dominant factors that influence the design.

The supply chain design to be addressed in this paper is highly integrated by considering sourcing, assembling, production, and final distribution in the same model. We briefly review below such global supply chain design models. A highly integrated model for designing global supply chain for Digital Equipment Corporation was developed in [10]. A mixed integer program was proposed that minimizes fixed and variable production costs, inventory costs, and distribution expenses, including transportation, taxes, and duty drawbacks. The model solves supply chain problems that involve multiple products, production stages, time periods, and transportation modes. The model links multiple supply tiers by the bill of material, and solves for the optimal solution over both production and distribution segments of the supply chain. The model proposed in the next section also links various activities in a fashion similar to bill of materials. The model in [10] also considers time measured as the number of days needed for production and for transit on each link in the supply chain, weighted by the number of units processed or shipped on the link. The objective is a weighted combination of total cost and overall supply chain response time.

In [11] a global supply chain model was developed to address design problems relating to a multinational corporation that outsources some but not all of its production to supplier facilities. This model simultaneously selects facility locations, computes flows between facilities, sets transfer prices, and allocates transportation costs to either the shipper or the receiver to maximize after-tax profits across multiple tiers in the supply chain. The resulting optimization problem is non-convex. In [12], the global supply chain design problem considered has a choice of foreign direct investment or outsourcing at each stage in the chain. A tax integrated approach is used and the resulting model is a mixed integer program.

The orchestration is also studied in the domain of logistics as global trade orchestrator - one who can orchestrate the many activities, dependencies, and relationships across a global logistics network [13]. Such players were called as integrated knowledge-based logistics providers in [14]. These new value players will dominate the supply chain, outsource non-core capabilities to contract manufacturers and third party logistics providers, and take control of the supply chain decision process. Optimization tools were proposed in [14] to manage some decision problems faced while synchronizing various activities across the network.

As exchange rate is not a major concern in this work we do not review literature related to it. Further we considered only integrated models. For a more detailed review on global supply chain design, see [15], [16]. An excellent textbook for methodologies and real cases is [9]. Another source devoted to global production network design is [17].

\section{THE MOdeL}

The model is progressively presented in this section, starting with problem definition, followed by notation and the mixed integer program.

\section{A. Problem definition}

The orchestrator receives an order for a quantity $D E M A N D$ of a certain product from customer $C$. The qualitative features and the delivery time are fixed and known. The orchestrator has to deliver the required quantity, with each unit adhering to the stated requirements, with 


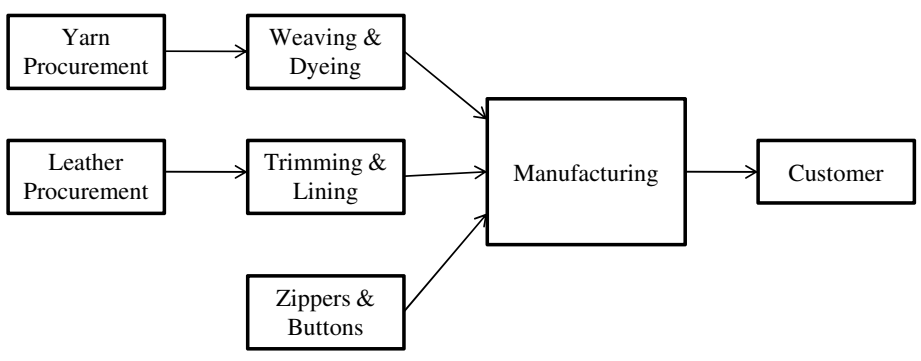

Fig. 1. Network of activities to be orchestrated

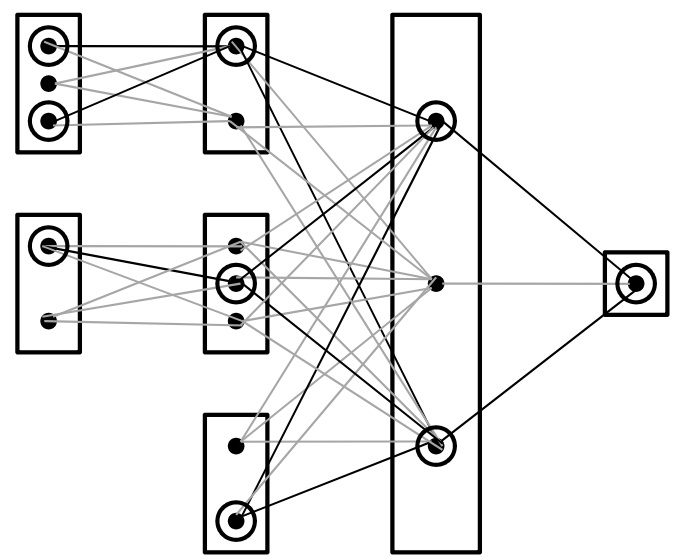

Fig. 2. Multi-layer network flow optimization minimal cost, and at the stipulated time. The orchestrator firstly disaggregates the entire manufacturing process into $N$ individual indivisible activities. Figure 1 shows a typical set of activities for producing a apparel like jacket. The activities are not exactly the same as the stages or echleons or tiers in supply chains, which are usually categorized based on inventory requirements. The activity is here defined as a indivisible service that has the choice of being provided by different service providers. The resulting network is a directed tree with activities as nodes and the flow of materials directed from the leaves to the root. Each leaf activity depends on no other activity and the root activity, labeled as $N$, is the delivery of the finished product to the customer C.

The leaf activities are usually procurement of raw materials or sub-assemblies. Each of the intermediate activities require inputs from the immediate predecessors. The linear combination of the inputs plus the local additions creates one unit of output in an activity. For example, in a shirt production activity the inputs eight buttons, one pocket, one collar, two sleeves, and two brand labels stitched together results in one shirt. There is only one successor to each activity (except for root with none) and at least one predecessor for activity (except for leaves with none). Thus the network has information regarding the precedence in activities and the recipe for each activity. One can obtain the above network from the bill of materials. For a product like PC or toys, the network could be complex with more activities and many different ways of combining them.

Each activity has the option of being single sourced or multi-sourced. For multi-sourcing, the orchestrator can restrict the number of service providers. Each activity also has a time duration, within which it has to be completed. The time requirements are mandatory constraints with no option for different service providers accomplishing the same activity on different cycle times. The directed edge from an activity to its successor also depicts the transportation of goods. We assume that there are no alternate choices of service providers for transporting. This assumption is induced from non-availability of time choice. Based on the time constraints, the shipping mode between any two service providers of adjacent activities will be fixed and hence the cost of transportation.

With the above network, the orchestrator shortlists appropriate service providers for each activity from its network of suppliers and based on its knowledge about their capabilities. They are chosen such that they closely match the customer's requirements in terms of quality and price. The individual activity requirements are then communicated to the respective shortlisted service providers for invitation of bids. A bid for an activity from the service provider consists of the price that he will charge for accomplishing the activity and supply quantity range (applicable for multi-sourcing activities). As lead time is a mandatory constraint, there is no negotiation in that dimension. The quality issues have to dealt with by the orchestrator by making quality checks during the process. This is one of the features that distinguishes the orchestrator from a B2B matching agent from electronic marketplaces that can merely match the service providers. Once the bids are obtained, the optimization problem faced by the orchestrator is to choose the service provider for each activity taking into various constraints.

Figure 2 shows the respective multi-layer network flow optimization of the network of figure 1. Each activity has alternate choices of service providers shown as dark dots. A feasible solution (network) is shown with circles around dots and dark lines on the links. The cost of the network is the total cost of procuring the service at each activity, transporting between different activities, and coordinating the entire network. In the following, we propose the mixed integer model for solving the above multi-layer network optimization problem. 


\section{B. Indices and Index Sets}

$\begin{array}{ll}i, j \in \mathcal{A} & \text { Activities, }|\mathcal{A}|=N ; \text { Root is labeled } N \\ p, l, m & \text { Service providers } \\ (i, l) \in \mathcal{P} \mathcal{A}_{i} & \text { Service providers for activity } i \\ c, n \in \mathcal{N} & \text { Nation groups } \\ (i, l) \in \mathcal{P} \mathcal{N}_{n} & \text { Service providers belonging to nation } \\ & \text { group } n \\ \mathcal{F} \mathcal{A} \quad(i \quad \notin \quad \text { Set of activities with no inputs (leaves) } \\ \mathcal{I} \mathcal{N}_{i}, \quad \text { Set of activities that provide direct in- } \\ \mathcal{F} \mathcal{A}) & \text { puts to } i \text { (predecessors) } \\ \hat{i} & \text { Activity that receives direct input from } \\ & i \text { (successor activity) }\end{array}$

C. Data

$\left[\underline{N S P}_{i}, \overline{N S P}_{i}\right]$ Range of admissible number of service providers for activity $i$

$P C O S T_{i l} \quad$ Unit cost of providing activity $i$ by service provider $(i, l) \in \mathcal{P} \mathcal{A}_{i}$ - received as bid

$\left[\underline{S U P}_{i l}, \overline{S U P}_{i l}\right]$ Range of units of activity $i$ available from $(i, l)$ - received as bid

$C C O S T_{i l} \quad$ Cost accrued to the orchestrator for coordinating with $(i, l)$ - quality checks, development, and training

$T C O S T_{i l j m}$ Unit cost of moving from $(i, l)$ to $\left(i \in \mathcal{I N}_{j}\right) \quad(j, m)$ - including transportation cost and duty

$I_{C O S T} T_{i j m}$ Cost accrued to the orchestrator for $\left(i \in \mathcal{I} \mathcal{N}_{j}\right) \quad$ coordinating the transfer of goods from $(i, l)$ to $(j, m)$ - customs handling, security clearance, interfacing with regulatory bodies

$D U T Y D_{c n} \quad$ Unit duty drawback for importing from nation group $c$ and exporting to nation group $n$ in different condition. For brevity, we assume all possible combinations.

$D E M A N D \quad$ Number of units demanded by the customer. The number of units required at for activity is converted appropriately converted to produce one unit of the required product. Hence, the demand for each activity is $D E M A N D$.

QUOTA $A_{n} \quad$ Available quota (units of the required product) for importing from nation group $n$. The quota is imposed on nation group which is the origin of the product, where it undergoes major transformation. We assume that this happens at activities that is the predecessor of activity $N$.
D. Decision Variables

$x_{i l} \in\{0,1\}$, Binary variable to select service provider $(i, l)$ for activity $i$

$y_{i l j m} \geq 0$, Units transferred from $(i, l)$ to $(j, m)(i \in$ $\mathcal{I} \mathcal{N}_{j}$ )

$z_{\text {iljm }} \in\{0,1\}$, Binary variable to decide whether $y_{i j l m}>0$

$d_{j l c n} \geq 0$, Units provided by $(j, l) \in \mathcal{P} \mathcal{A}_{j}$ that can avail duty drawback for importing from nation group $c$ and exporting to nation group $n$

\section{E. Constraints}

1) Sourcing: Constrain the number of service providers for each activity. For single sourcing at $i, \underline{N S P}_{i}=\overline{N S P}_{i}=$ 1.

$$
\underline{N S P}_{i} \leq \sum_{(i, l) \in \mathcal{P} \mathcal{A}_{i}} x_{i l} \leq \overline{N S P}_{i}, \forall i \in \mathcal{A} \backslash\{N\}
$$

2) Supply and demand:

$$
\begin{aligned}
& y_{i l \hat{i} m} \leq B i g M z_{i l \hat{i} m}, \\
& \forall i \in \mathcal{A} \backslash\{N\},(i, l) \in \mathcal{P} \mathcal{A}_{i},(\hat{i}, m) \in \mathcal{P} \mathcal{A}_{\hat{i}} \\
& \underline{S U P}_{i l} x_{i l} \leq \sum_{(\hat{i}, m) \in \mathcal{P} \mathcal{A}_{\hat{i}}} y_{i l \hat{i} m} \leq \overline{S U P}_{i l} x_{i l}, \\
& \forall(i, l) \in \mathcal{P} \mathcal{A}_{i}, \forall i \in \mathcal{A} \backslash\{N\} \\
& \sum_{(i, l) \in \mathcal{P} \mathcal{A}_{i} \sum_{(\hat{i}, m) \in \mathcal{P} \mathcal{A}_{\hat{i}}} y_{i l \hat{i} m}=D E M A N D, \quad \forall i \notin \mathcal{F} \mathcal{A}}
\end{aligned}
$$

3) Flow constraint for service provider:

$$
\begin{aligned}
& \sum_{i \in \mathcal{I N}_{j}} \sum_{(i, l) \in \mathcal{P} \mathcal{A}_{i}} y_{i l j m}=\left|\mathcal{I N}_{j}\right| \sum_{(\hat{j}, p) \in \mathcal{P} \mathcal{A}_{\hat{i}}} y_{j m \hat{j} p}, \\
& \forall j \notin \mathcal{F} \mathcal{A} \cup\{N\}
\end{aligned}
$$

4) Duty drawbacks: The duty drawback that is considered here is exporting the good in different condition. This also includes re-exporting to the nation group after value addition. The DUTY $D_{c n}$ appropriately captures per unit drawback for the above scenarios. For every service provider, we model the duty drawback variables as flows on a transportation problem. The sources are the nation groups from where the inputs are imported and the destinations are the nation groups where the goods are exported with value addition. The supply capacity for the sources and the demand at the destinations are appropriately calculated from the flow variables $\{y\}$.

$$
\begin{aligned}
& \sum_{n} d_{j m c n} \leq \sum_{i \in \mathcal{I} \mathcal{N}_{j}} \sum_{(i, l) \in \mathcal{P} \mathcal{N}_{c} \cap \mathcal{P} \mathcal{A}_{i}} y_{i l j m}, \\
& \forall j \notin \mathcal{F} \mathcal{A} \cup\{N\}, \forall c \in \mathcal{N} \\
& \sum_{c} d_{j m c n} \leq \sum_{(\hat{j}, p) \in \mathcal{P} \mathcal{N}_{n} \cap \mathcal{P} \mathcal{A}_{\hat{j}}} y_{j m \hat{j} p}, \\
& \forall j \notin \mathcal{F} \mathcal{A} \cup\{N\}, \forall n \in \mathcal{N}
\end{aligned}
$$

5) Import quotas:

$$
\sum_{i \in \mathcal{I} \mathcal{N}_{N}} \sum_{(i, l) \in \mathcal{P} \mathcal{A}_{i} \cap \mathcal{P N}_{n}} y_{i l C N} \leq \text { QUOT } A_{n}, \forall n \in \mathcal{N}
$$




\section{F. Objective}

The objective is to minimize the overall cost of orchestration. This includes the fixed costs of coordinating each selected supplier, coordinating each selected link in the network, and variable production and shipping costs.

$$
\begin{aligned}
& \min \sum_{i \in \mathcal{A} \backslash\{N\}} \sum_{(i, l) \in \mathcal{P} \mathcal{A}_{i}} \operatorname{CCOST}_{i l} x_{i l} \\
& +\sum_{i \in \mathcal{A} \backslash\{N\}} \sum_{(i, l) \in \mathcal{P} \mathcal{A}_{i}} \operatorname{PCOST}_{i l} \sum_{(\hat{i}, m) \in \mathcal{P} \mathcal{A}_{\hat{\imath}}} y_{i l \hat{i} m} \\
& +\sum_{i \in \mathcal{A} \backslash\{N\}} \sum_{(i, l) \in \mathcal{P} \mathcal{A}_{i}} \sum_{(\hat{i}, m) \in \mathcal{P A}_{\hat{i}}} \operatorname{TCOST}_{i l \hat{i} m} y_{i l \hat{i} m} \\
& +\sum_{i \in \mathcal{A} \backslash\{N\}} \sum_{(i, l) \in \mathcal{P} \mathcal{A}_{i}} \sum_{(\hat{i}, m) \in \mathcal{P} \mathcal{A}_{\hat{i}}} I C O S T_{i l \hat{i} m} z_{i l \hat{i} m} \\
& -\sum_{c \in \mathcal{N}} \sum_{n \in \mathcal{N}} D U T Y D_{c n} \sum_{j \in \mathcal{A} \backslash\{N\}} \sum_{(j, m) \in \mathcal{P} \mathcal{A}_{j}} d_{j m c n} \\
& \text { V. Final Notes }
\end{aligned}
$$

Orchestration is increasingly becoming a popular model for businness innovation and service delivery. Our particular focus was on orchestrators who own no hard capacities. Their main assets are the relationship with a large pool of service providers and the knowledge about the diverse production factors, trade cultures, and goverment regulations. Upon arrival of a customer order, a build-to-order strategy is used to design a supply chain tailored to manufacture the demanded product. We proposed a mixed integer program to optimally design the supply chain taking into account various factors related to global manufacturing systems. We address below the potential research problems in this domain.

\section{A. Algorithm}

The proposed mixed integer program can be solved using commercial optimization packages. Even if one expects around few alternative service providers for each activity, the problem can become computationally demanding with too many interdependent decision variables. Some of the binary variables (especially from $\{z\}$ ) can become redundant for certain problem instances and also the duty drawback variables. However, the presence of such interrelationships can even render the sophisticated commercial packages clueless while using branch and bound algorithms. Exploiting the several known structures in the problem (like network flow and transportation) and developing branch and cut algorithms can tame the computational demands.

\section{B. Lead times and transportation modes}

We assumed for simplicity fixed lead times and fixed transportation modes. Allowing choices for transportation modes can possibly decrease cost. For example, a low cost service provider may have available capacity immediately which may require expedited shipping from the input activities and can trade-off the shipping cost with low production cost. The complexity of the problem, however, increases in the dimension of time factor and requires scheduling of activities in tandem with network flow.

\section{Service provider disruption}

One of the concerns is the disruption of a service provider during the execution. The orchestrator should be able to shift to another alternative with least cost and also with disrupting the already established network of service providers. Stochastic programming tools can help in handling such uncertainties.

\section{Developing new service providers}

The proposed model chooses service providers based by minimizing coordination costs (one of the terms in the objective function). This discourages choosing new service providers as the coordination costs are high. However, for the orchestrator, it is required to develop and accommodate new service providers. The model should be able trade-off this qualitative feature with the quantitative coordination cost.

\section{E. Combinatorial bidding}

Coordination cost for a service provider is a fixed cost. The model assumed no common service provider across different activities. This can be included with certain inequalities. The interesting extension is to allow combinatorial bidding - where a service provider can bid for a combination of activities and possibly quote a reduced price for due to economies of scope. Combinatorial bidding has been successfully employed for many industrial procurements [18]. However, the computational demands on the optimization problem increases significantly and it is an interesting research problem.

\section{F. Muliple products}

The customer demand considered is this paper is for multiple units of a single product. If there is a demand for multiple products that share no common activities, then they can be treated as different customer orders. However, for multiple products that share certain common activities, merging them into a single network can achieve economies of scale, but with added complexity in design and optimization.

\section{G. Service providers interactions}

Each customer order is treated as a project and a set of service providers are assembled and managed to execute the project. The modeling of interactions between service providers of adjacent activities can provide useful information regarding the compatibility of the service providers. With better compatibility between the service providers, the orchestrators can gain with less coordination costs.

\section{H. Developing competitors}

The interactions between service providers can also lead to competition threat. One of the greates risks in orchestration is the partner becoming the competitor. With the repeated interactions and good compatability, a strategically colluded subset of service providers can have the same end-to-end visibility as the orchestrator. If such a coalition decides to 
move up the value chain, then it is a potential competitor to the orchestrator.

\section{Network restructuring}

As noted earlier, an important asset to the orchestrator is the relationships with the service providers. The loosely coupled network of service providers needs to be restrucured - expanded, pruned, repositioned - in repsonse to the market conditions. There are interesting decision problems related to how much to invest in developing new service providers, which suppliers should be upgraded with new technology, etc.

\section{REFERENCES}

[1] J. P. Andrew, H. L. Sirkin, and J. Butman, Payback: Reaping the Rewards of Innovation. Boston, Massachusetts: Harvard Business Press, 2006.

[2] C. Dhanraj and A. Pharke, "Orchestrating innovation networks," Academy of Management Review, vol. 31, no. 3, pp. 659-669, 2006.

[3] A. Ordanni, K. L. Kraemer, and J. Dedrick, "Medion: the retail "Orchestrator" in the computer industry," Personal Computing Industry Center, Tech. Rep., 2006.

[4] J. O'Connell, "Li \& Fung (Trading) Ltd," Harvard Business School, Teaching Case 9-396-075, 2006.

[5] W. Fung and Y. Wind, Competing in a Flat World: Building Enterprises for a Borderless World. Upper Saddle River, NJ: Wharton School of Publishing, 2007.

[6] J. Magretta, "Fast, global, and entrepreneurial: Supply chain management, Hong Kong style. An interview with Victor Fung," Harvard Business Review, pp. 103-114, September-October 1998.

[7] S. Chopra and P. Meindl, Supply Chain Management: Strategy, Planning and Operations. Upper Saddle River, NJ: Prentice Hall, 2004.

[8] Z. M. Mohamed, "An integrated production-distribution model for a multi-national company operating under varying exchange rates," International Journal of Production Economics, vol. 58, no. 1, pp. 81-92, 1999.

[9] P.-P. Dornier, R. Ernst, M. Fender, and P. Kouvelis, Global Operations and logistics: Text and cases. New York: John Wiley \& Sons, 2002.

[10] B. C. Arntzen, G. G. Brown, T. P. Harrison, and L. L. Trafton, "Global supply chain management at Digital Equipment Corporation," Interfaces, vol. 25, no. 1, pp. 69-93, 1995.

[11] C. J. Vidal and M. Goetschalckx, "A global supply chain model with transfer pricing and transportation cost allocation," European Journal of Operational Research, vol. 129, no. 1, pp. 134-158, 2001.

[12] K. Balaji and N. Viswanadham, "A tax integrated approach for global supply chain network planning," IEEE Transactions on Automation Science and Engineering, vol. 5, no. 4, pp. 587-596, 2008.

[13] Q. Wedan, "Transforming global logistics for strategic advantage in emerging markets," IBM Global Business Services," Whiten Paper, June 2006

[14] N. Viswanadham and R. Gaonkar, "A conceptual and analytical framework for management of integrated knowledge based logistics providers," International Journal of Logistics Systems and Management, vol. 5, no. 1, pp. 191-209, 2009.

[15] C. J. Vidal and M. Goetschalckx, "Strategic production - distribution models: A critical review with emphasis on global supply chain models," European Journal of Operational Research, vol. 98, no. 1, pp. 1-18, 1997.

[16] M. J. Meixell and V. B. Gargeya, "Global supply chain design: A literature review and critique," Transportation Research, vol. 41, pp. 531-550, 2005.

[17] R. Hubner, Strategic Supply Chain Management in Process Industries. Berlin: Springer, 2007.

[18] P. Cramton, Y. Shoham, and R. Steinberg, Combinatorial Auctions. Cambridge: MIT Press, 2005. 